\title{
Conductas, actitudes y prácticas de la madre o \\ cuidador en el manejo de la enfermedad diarreica aguda en menores de cinco años
}

ISSN 1794-9831

E-ISSN 2322-7028

Vol. 12 No. 2

Jul - Dic 2015

Cúcuta, Colombia

María del Pilar Gallardo-Lizarazo*

Objetivo: determinar las creencias de la madre y del cuidador sobre el manejo de la enfermedad diarreica aguda en menores de 5 años de edad en la ciudad de Bucaramanga en el año 2015. Materiales y Métodos: la investigación se enmarca en el paradigma cuantitativo mediante el desarrollo de estudio descriptivo de corte transversal; se encuestaron 200 madres o cuidadores de menores de cinco años en la ciudad de Bucaramanga, seleccionados mediante muestreo aleatorio estratificado con afijación proporcional, confiabilidad 95\% y margen de error de 7\%. Para la recolección de la información se empleó una encuesta elaborada por la autora con validación estadística y por expertos. Resultados: se obtiene información sobre conocimientos y tradiciones acerca del manejo de la diarrea y la identificación de los signos de deshidratación por parte de los cuidadores, que son analizados en comparación con sus características sociales y culturales. Conclusiones: en Bucaramanga, madres y cuidadores de menores de cinco años, continúan la tradición familiar en lo referente a identificación de signos de deshidratación y costumbres en el manejo de la diarrea; la mayoría acude a consulta médica para su tratamiento; sin embargo, se encuentra un alto porcentaje que también recurre al tegua-sobandero. Para el manejo de la diarrea, las madres o cuidadores suministran caldos a los menores, aumentan la cantidad de líquidos, les administran sales de rehidratación oral y jugo de guayaba. Se encuentran diferencias acerca de las costumbres y tradiciones entre estratos socioeconómicos y niveles de escolaridad.

PALABRAS CLAVE: costumbres, cuidadores, diarrea infantil ${ }^{* *}$.

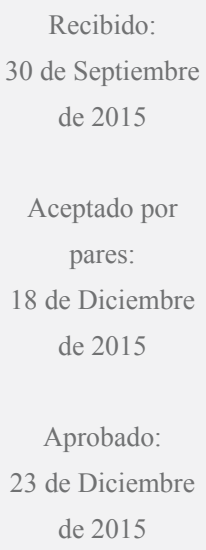

Médico. Médico General Consulta Externa. Sanitas E.P.S. Estudiante Especialización en Epidemiología. Bucaramanga, Colombia. Correo electrónico: mapygallar@ hotmail.com

** Descriptores en Ciencias de la Salud (DeCS), en la página $\mathrm{http}: / /$ decs.bvs.br/E/ homepagee.htm de la Biblioteca virtual en salud del proyecto BIREME, de la Organización Mundial de la Salud y de la Organización Panamericana de Salud. 


\section{Comportamentos, atitudes e práticas da mãe ou responsável na gestão da diarreia aguda em crianças menores de cinco anos}

ISSN 1794-9831 E-ISSN 2322-7028

Vol. 12 No. 2

Jul - Dic 2015

Cúcuta, Colombia

\section{ABSTRACT}

Objetivo: para determinar as crenças de mães e cuidadores sobre a gestão da diarreia aguda em crianças menores de 5 anos na cidade de Bucaramanga em 2015. Materiais e Métodos: a pesquisa é parte do paradigma quantitativo por desenvolvimento de estudo descritivo transversal; 200 mães ou cuidadores de crianças menores de cinco anos foram pesquisados na cidade de Bucaramanga, selecionadas por amostragem aleatória estratificada com alocação proporcional, confiabilidade e margem de $95 \%$ de erro de $7 \%$. Para pesquisa de coleta de dados realizada pelo autor com validação estatística e os peritos que ele estava acostumado. Resultados: informações sobre o conhecimento e tradições sobre a gestão de diarreia e identificar sinais de desidratação por cuidadores, que são analisadas em comparação com as suas características sociais e culturais é obtido. Conclusões: em Bucaramanga, mães e cuidadores de crianças menores de cinco anos, continuar a tradição da família quando se trata de verificar se há sinais de desidratação e alfândegas na gestão da diarreia; mais ir ver o seu médico para tratamento; no entanto, há uma alta porcentagem que também se baseia Tegua-sobandero. Para a gestão de diarreia, mães ou cuidadores fornecem vinhos a menores, aumentar a quantidade de fluidos administrados los ORS e suco de goiaba. diferenças sobre os costumes e tradições entre os níveis socioeconômicos e educacionais são.

PALAVRAS-CHAVE: costumes, cuidadores, diarreia infantil. 
ISSN 1794-9831

E-ISSN 2322-7028

Vol. 12 No. 2

Jul - Dic 2015

Cúcuta, Colombia

\section{INTRODUCCIÓN}

A nivel mundial ha tomado gran importancia identificar y tratar las deposiciones diarreicas con adecuada hidratación para prevenir futuras complicaciones; puesto que esta enfermedad se encuentra establecida como la segunda causa de muerte, afectando principalmente a los menores de cinco años, sin desconocer los demás grupos cronológicos y sin distinguir características étnicas. Cada niño menor de 5 años de edad presenta un promedio de tres episodios anuales de diarrea aguda (1) y, según la Organización Panamericana de la Salud (OPS), se estima que más de un millón y medio de niños menores de cinco años mueren por diarrea cada año y la mayoría de esas muertes ocurren por deshidratación (2).

Por otra parte, el Instituto Nacional de Salud indica que en Colombia más de 250000 niños mueren antes de los cinco años por enfermedades que podrían prevenirse fácilmente. Estas muertes ocurren principalmente por diarrea, neumonía, desnutrición, y otras enfermedades prevenibles por vacunación, siendo éstas la causa del $60 \%$ al $80 \%$ de las consultas pediátricas en los servicios de salud y del $40 \%$ al 50 $\%$ de las hospitalizaciones de niños menores de cinco años (3).

La mortalidad por enfermedad diarreica aguda (EDA) ocupa uno de los primeros lugares en la mortalidad de los menores de cinco años, especialmente en los municipios con mayor proporción de necesidades básicas insatisfechas y mala calidad de agua para consumo humano (4). La incidencia de morbilidad por EDA en el país, para el año 2014, es de 60,05 por cada mil habitantes, más alta en comparación con la de 2013 que fue de 57,3 y de 54,76 en 2012 (5).

Existen diferentes trabajos sobre EDA y las creencias de los cuidadores de menores. En Ica, Perú (6), se encontró que, en promedio, las madres encuestadas tenían la creencia de que en deposiciones diarreicas debían suspender la lactancia materna y que los parásitos son la principal causa de la presencia de esta enfermedad, por lo cual era necesario el uso frecuente de antibióticos y antiparasitarios.

En México (7) se encontró afinidad entre EDA como factor de defunción y la falta de identificación de los signos de peligro; sin embargo, el estudio muestra que la atención que se brindó en el hogar fue apropiada. Otro estudio encontró que en episodios de EDA la deshidratación es tratada como un signo más de la enfermedad y no como la pérdida de líquidos corporales que ocasiona una complicación (8).

En Mozambique se indagaron los signos de deshidratación percibidos por la madre en los episodios de diarrea aguda en los niños, encontrando como los más referenciados: pérdida de peso, debilidad, disminución de apetito y también se encontró el no conocimiento de cómo preparar sueros caseros (9).

En España se realizó un estudio descriptivo donde se encontró que el $20 \%$ de las madres no tiene claridad en la descripción y conocimiento de un episodio diarreico y que la cuarta parte de las entrevistadas, al evidenciar un aumento en las deposiciones en el menor, lo sometían a periodos prolongados de ayuno usando en esa misma forma las fórmulas de rehidratación (10).

En Etiopía, Yalew (11) realiza un estudio cualitativo sobre las percepciones hacia las causas y tratamiento de la diarrea infantil por parte del cuidador y de la comunidad y su gestión en el distrito Assosa, en el cual se evidencia que la comprensión de esta enfermedad es esencial para planificar medidas y programas eficaces de prevención y control. Encuentra que muchos de los participantes perciben la diarrea como una de las tres enfermedades más comunes en la infancia, al tiempo que los cuidadores perciben la higiene personal inadecuada y la falta de saneamiento ambiental como las principales causas de la diarrea infantil, indicando que muchos de los cuidadores tenían la correcta comprensión de las causas de esta enfermedad.

En el mismo estudio (11) algunos participantes asociaron la diarrea infantil con erupción de dientes de leche, chupar la leche materna caliente, exposición al calor y el clima, entre otros. El trabajo brinda información a los funcionarios de salud y otras partes interesadas en desarrollar mejores estrategias para evitar este tipo de errores de percepción.

Bruce et al. (12) identifican las barreras en la búsqueda de atención a niños con neumonía y diarrea de zonas rurales de Guatemala. En la investigación aplican análisis multivariante para determinar factores asociados a la percepción de la madre sobre 
las señales de peligro y la percepción de la gravedad de la enfermedad, encontrándose como determinantes la atención en el cuidado formal aunado al desarrollo social y el nivel de educación de la madre.

Arvelo et al. (13) describen los conocimientos y las ideas que tienen las personas que prestan atención de salud en el sector convencional, el sector no convencional y los cuidadores de la comunidad con respecto al uso de las soluciones de rehidratación oral en el tratamiento de las enfermedades diarreicas, en el departamento guatemalteco de Santa Rosa, encontrando que los participantes en el estudio atribuyen la causa de los episodios de diarrea en los niños a dolencias culturalmente aceptadas o populares -empacho, cuajo y varillas-, que tratan principalmente los curanderos.

Asimismo, encuentra deficiencias en los conocimientos acerca de la deshidratación como una manifestación de la diarrea y su tratamiento, incluido el uso de las soluciones de rehidratación oral y la necesidad de continuar la alimentación durante los episodios diarreicos. Los cuidadores consideraron las soluciones de rehidratación embotelladas o preparadas y los medicamentos antidiarreicos de venta libre, que son más costosos, como mejores opciones que las soluciones de rehidratación oral para el tratamiento de la diarrea (13).

Pantenburg et al. (14) investigaron en Lima sobre las prácticas y percepciones de los cuidadores sobre la alimentación de los niños pequeños durante la diarrea, descubriendo percepciones erróneas sobre las prácticas en alimentación de los menores durante episodios de esta enfermedad, pues se identificó que un alto porcentaje de los cuidadores suspendía la alimentación normal o daba menos comida. En particular, la tendencia era a retener carnes, leche y huevos y, en menor proporción, a suspender frutas y verduras.

Los investigadores realizan una intervención educativa piloto para mejorar la alimentación durante la diarrea $y$, mediante encuesta de seguimiento aplicada tres meses después, encontraron un cambio de actitud en el sentido de que ninguno de los participantes recomendaría la retención de alimentos. El estudio proporciona evidencia de que las prácticas y percepciones de los cuidadores pueden ser mejoradas mediante una breve intervención educativa (14).
Carter et al. (15) afirman que las prácticas nocivas en el manejo de la diarrea infantil están asociadas con resultados negativos en su salud. Estas prácticas incluyen la restricción de líquidos, la leche materna y/o la ingesta de alimentos durante los episodios de diarrea y el uso incorrecto de los medicamentos modernos.

Carter et al.(15) revisaron 114 artículos publicados en idioma inglés desde 1990 para evaluar la prevalencia documentada de prácticas, creencias, motivaciones y factores contextuales relacionados en países con ingresos bajos y medios. Encontraron que en el 69 $\%$ de los artículos se describe al menos una práctica perjudicial y en el $31 \%$ se informa sobre creencias y motivaciones nocivas.

Además, los médicos informaron que las prácticas en el manejo de la diarrea se basan en el consejo de otros -trabajadores de la salud, familiares y miembros de la comunidad-, así como en sus propias observaciones o comprensión de la eficacia de ciertos tratamientos para la enfermedad. Por otro lado, encuentran que en algunos países con alta mortalidad por diarrea hay evidencia de prácticas nocivas, las cuales pueden reducir el correcto manejo de las enfermedades diarreicas en menores, hecho que conduce a fracasos en su tratamiento y a elevar las tasas de mortalidad por EDA en la población infantil (15).

Kung'u et al. (16) utilizaron entrevistas semiestructuradas para recopilar información sobre el conocimiento, actitudes y prácticas relacionadas con la diarrea en cuidadores de menores de cinco años de edad y en trabajadores de la salud en Nigeria. En su estudio, hallaron que casi todos los trabajadores de la salud mencionan correctamente la dosis de zinc, mientras que sólo un poco más de la mitad de los cuidadores declararon correctamente la preparación de sales de rehidratación oral.

También encuentran que el porcentaje de cuidadores con conocimientos sobre el tratamiento de la diarrea aumentó de $46 \%$ en 2010 a $71 \%$ en 2011. Mediante intervención se logró el aumento significativo en el conocimiento en los cuidadores sobre el manejo de la diarrea. Concluyen que existen oportunidades para que los cuidadores aprendan sobre el uso de sales de rehidratación oral para el tratamiento de la enfermedad (16).
ISSN 1794-9831

E-ISSN 2322-7028

Vol. 12 No. 2

Jul - Dic 2015

Cúcuta, Colombia 
ISSN 1794-9831

E-ISSN 2322-7028

Vol. 12 No. 2

Jul - Dic 2015

Cúcuta, Colombia
En la India, Lamberti et al. (17) desarrollan un estudio para determinar la influencia de la severidad del episodio en la búsqueda de atención y tratamiento de la diarrea por parte de los cuidadores de niños entre 2 y 59 meses de edad. Utilizando el análisis de regresión mostraron una tendencia de mayor severidad en episodios menos recientes en comparación con los más recientes así como también una fuerte correlación entre la búsqueda de atención y la deshidratación, fiebre, vómitos y una mayor frecuencia de deposiciones y la duración. El tratamiento con sales de rehidratación oral se asoció con la deshidratación, vómitos y una mayor frecuencia de las deposiciones. También encuentran que el tratamiento recibido por los niños en el sector público es de menor calidad que el recibido por los del sector privado.

En Colombia, cada cinco años, Profamilia realiza la Encuesta Nacional de Demografía y Salud. En el año 2000 se evaluaron los conocimientos sobre el tratamiento de rehidratación oral, evidenciando que el $92 \%$ de las madres con nacidos vivos en los últimos 5 años conocían acerca del manejo de hidratación en las deposiciones diarreicas. Este porcentaje aumentó con la edad de la madre, el nivel de educación y fue mucho mayor en la zona urbana (18).

En la encuesta del año 2012 se encontró que el 88 $\%$ de las madres conocían las sales de rehidratación oral, el $72 \%$ de esas madres eran menores de 20 años, el $74 \%$ de las mujeres no tenían educación y el $96 \%$ de las mujeres con educación universitaria tenían conocimiento sobre estas sales. Las mujeres aumentaban su conocimiento con el nivel de riqueza, pasando de $80 \%$ entre las de índice de riqueza más bajo a $95 \%$ entre las de índice de riqueza más alto (19).

Es por esto que se han identificado estrategias para educar a las madres o cuidadores sobre el manejo adecuado en casa y centros de salud de primer nivel, buscando la oportuna atención y realización de las convenientes prácticas de hidratación. Por ello se creó la estrategia de Atención Integrada de las Enfermedades Prevalentes de la Infancia (AIEPI) que está encaminada a disminuir la mortalidad ocasionada por las enfermedades prevalentes de la infancia, específicamente en menores de 5 años.

AIEPI es una estrategia que busca disminuir la mortalidad por enfermedades prevalentes en menores de cinco años educando al médico tratante y al entorno familiar. En la EDA, que es una de las principales causas de consulta en el servicio de urgencias de pediatría, las actividades se encaminan a promocionar los conocimientos maternos sobre signos de peligro, el manejo del niño con EDA en el hogar, la búsqueda oportuna de atención y las prácticas adecuadas de hidratación, alimentación y enseñanza sobre signos para re consultar cuando resulte necesario (20).

Lo primordial para el cuidado de un niño que tiene diarrea es mantenerlo hidratado, dándole agua, leche materna -si aún la consume- y los alimentos que comúnmente recibe a diario, pero también es importante que madres y cuidadores estén atentos y capacitados en la identificación de señales de deshidratación.

En el país se encuentran diferentes comportamientos asociados al cuidado del menor ante la presencia de la diarrea. Tal es el caso del estudio realizado por Rodríguez et al. (21), quienes investigaron sobre las prácticas y creencias culturales acerca del cuidado de niños menores de un año en un grupo de madres de Chocontá, encontrando mitos como poner esparadrapo o acudir a un sobandero que realice masajes en el abdomen y rezos para curar la diarrea.

En un estudio realizado en Cúcuta (22) se encontró que la mayoría de los cuidadores de los menores de cinco años tiene un bajo nivel socioeconómico y la limitación para adquirir una calidad de vida que permita el desarrollo y crecimiento sano de los niños, además de aplicar cuidados especiales de acuerdo a sus creencias y cultura; sin embargo, se encuentra que así como hay cuidadores que poseen conocimientos adecuados sobre la inclusión oportuna de la alimentación complementaria, existen determinantes de riesgo, en otros casos, por la ausencia de conocimientos acertados sobre la alimentación complementaria y micronutrientes que confluyen en el inicio temprano de esa alimentación complementaria, con el objetivo de que el menor, al probar los alimentos que consume la familia, se vuelva más fuerte frente a las infecciones o se le cure el estómago, todo lo anterior enmarcado en un ciclo de creencias que han adoptado los cuidadores desde sus ancestros.

Dentro de las prácticas de riesgo se pudo detectar que los cuidadores suministran a los niños/as alimentos 
diferentes al grupo que debe consumir de acuerdo a su edad, en ocasiones mayores para el rango de edad y en otras de manera deficiente. Otra práctica de riesgo es el abandono temprano de la lactancia materna -seis meses de edad- al considerar que el menor ya consume otros alimentos que suplen sus necesidades para el crecimiento (22).

Al respecto, en Bucaramanga se han realizado diversos estudios, uno de los cuales fue desarrollado por la Escuela de Medicina de la Universidad Industrial de Santander en coordinación con la Secretaría de Salud Municipal (23), en el año 2012, enfocado al análisis de la situación de salud. Este trabajo reveló que las diez primeras causas de consulta externa en el grupo de 0 a 4 años correspondieron principalmente a enfermedades infecciosas en un $79.8 \%$ en 2011 ; en tercer lugar se ubicó la diarrea y la gastroenteritis de presunto origen infeccioso. El informe hace un llamado a trabajar más en brindar servicios centrados en la promoción de la salud que en el cuidado de las enfermedades.

Uribe et al. (24) descubrieron, en los aislamientos de patógenos comunes asociados con enfermedad diarreica aguda en menores de 5 años, los rotavirus -que tienen un importante papel en el municipio de Bucaramanga, como principales agentes patógenos aislados asociados con enfermedad diarreica aguda en niños menores de 5 años-. El estudio se elaboró con el propósito de documentar los gérmenes aislados más frecuentemente $\mathrm{y}$, por consiguiente, orientar o fortalecer la conducta clínica y de salud pública en esta población.

En Bucaramanga se elaboró otro estudio (25) para describir las representaciones sociales que tienen los cuidadores de los niños menores de cinco años sobre la EDA, encontrándose que el $73 \%$ de cuidadoras eran madres con rango de edad entre 14 y 71 años. El $3 \%$ identificó componentes de definición de diarrea. Un tercio de cuidadores atribuyó diarrea a descuajaduras, mal de ojo y brujería. El 70 \% reconoce la duración de síntomas por más de 24 horas como criterio para consultar a una institución de salud, $30 \%$ fiebre y $10 \%$ deshidratación. El $83 \%$ conoce soluciones comerciales de rehidratación y el $63 \%$ identifica las sales de rehidratación oral, aunque sólo el $46 \%$ las ha usado. E1 $63.82 \%$ usa remedios caseros (25). También hay gente que se asusta y recurre a un empírico, quien "soba" o administra medicamentos inapropiados al niño.
En consecuencia, de conformidad con la estrategia AIEPI que busca mejorar la calidad y la atención de los menores en los servicios de salud, además de reducir la morbilidad y mortalidad en menores de cinco años especialmente en las enfermedades más prevalentes -y que con referencia al manejo de la diarrea propone incentivar que los alimentos y depósitos de agua se mantengan tapados, fomentar el lavado de manos, promover el uso adecuado de agua en la preparación de alimentos y en la higiene personal, entre otros-resulta importante establecer las creencias, conductas y actitudes que tienen las madres o cuidadores de menores de 5 años en este aspecto, puesto que es un tema poco explicado y/o comprendido a nivel mundial y de Latinoamérica.

\section{MATERIALES Y MÉTODOS}

La presente investigación determina, mediante un estudio descriptivo de corte transversal, las creencias maternas o de cuidadores sobre el manejo de EDA en niños menores de 5 años de edad en la ciudad de Bucaramanga, en el año 2015, al tiempo que describe sus características socio-demográficas y establece relaciones entre sus creencias y costumbres y los factores sociales y culturales asociados.

El estudio se enmarca en los lineamientos de Hernández et al. (26), Villa et al. (27), Bonita et al. (28), Rothman K. (29) y Hurtado J. (30). La validez del diseño expresado como tipo de estudio para la investigación propuesta se sintetiza en los aspectos de validez interna, externa y conceptual, en lo referido al enfoque cualitativo expresado por Hurtado y Toro (31).

La población objeto de estudio está constituida por las madres o cuidadores de niños menores de 5 años en la ciudad de Bucaramanga. Ante la inexistencia de registros que permitieran identificar el marco muestral o universo, se recurrió a una aproximación indirecta consistente en la estimación a partir de los niños menores de cinco años.

Con esa premisa y con base en la proyección del Departamento Nacional de Estadística (DANE) para el año 2015 sobre la población en la ciudad de Bucaramanga, en la que se indica un total de 34922 niños y niñas menores de cinco años, se infiere un número similar de cuidadores, quienes constituyen la población diana.
ISSN 1794-9831

E-ISSN 2322-7028

Vol. 12 No. 2

Jul - Dic 2015

Cúcuta, Colombia

Cuscuta, Colon

(⿸丆口 
ISSN 1794-9831

E-ISSN 2322-7028

Vol. 12 No. 2

Jul - Dic 2015

Cúcuta, Colombia
El diseño muestral se formalizó mediante el método aleatorio estratificado con afijación proporcional, determinado por los estratos socioeconómicos, dando el mismo peso a la variable de decisión en cada estrato por cuanto no se tiene aproximación inicial a este valor. Se estableció un nivel de confiabilidad de $95 \%$ con un error máximo admisible de $7 \%$ :

$$
n=\frac{34922}{1+\frac{0.07^{2} *(34922-1)}{1.96^{2} * 0.5 * 0.5}}=195
$$

Para el estudio se incluyeron 200 madres o cuidadores de Bucaramanga con niños menores de 5 años a cargo, quienes fueron contactados durante el primer semestre del año 2015 en las viviendas seleccionadas con base en la estratificación por predios, distribuidos en forma proporcional a la composición, por estratos socioeconómicos, de la población de la ciudad.

A continuación se seleccionaron al azar, en barrios de cada estrato, unas manzanas y, en cada una de ellas, viviendas, así: se inicia por la esquina noroeste de la manzana y se contacta una vivienda, se continúa en sentido de las manecillas del reloj, seleccionando cada tercera vivienda hasta encontrar cuatro viviendas por manzana seleccionada, con menores de cinco años. $\mathrm{Si}$ en una vivienda seleccionada no hay menores de cinco años o si no hay moradores, se salta a la siguiente vivienda a seleccionar, de conformidad con el procedimiento descrito.

En las viviendas en que se encontró un menor de cinco años se aplicó la encuesta a su cuidador principal. $\mathrm{Si}$ una vivienda seleccionada es multifamiliar o corresponde a un conjunto residencial, se selecciona una familia al azar y se continúa con el procedimiento establecido. Una vez cubierta toda una manzana se pasa a la siguiente, que ha sido seleccionada previamente al azar.

La distribución de las encuestas, acorde a lo referido, fue la siguiente: estrato uno: 18 encuestas $(9 \%)$; estrato dos: 32 (16\%); estrato tres: 60 (30\%); estrato cuatro: 64 (32\%); estrato cinco: $10(5 \%)$; y estrato seis: 16 encuestas $(8 \%)$.

Los criterios de inclusión fueron: madres o cuidadores de niños menores de 5 años. Los criterios de exclusión: madres o cuidadores de niños con patologías de base que alteren el comportamiento de la diarrea, tales como: intolerancia a la lactosa, síndrome de colon irritable, desnutrición ya establecida como diagnóstico previo, alteraciones neurológicas, alteración en la ingesta de carbohidratos, enfermedad de reflujo gastroesofágico, síndromes de inmunodeficiencia congénitos o adquiridos, e incluyendo niños que estén recibiendo complemento nutricional y madres de niños menores de 5 años con alguna de las siguientes características: su estado de salud impide el diligenciamiento de los formatos, se les identifica otro diagnóstico que excluyó o hizo poco probable el de Enfermedad Diarreica Aguda (EDA).

Las variables estudiadas se clasificaron así:

- Variables Dependientes: Creencia, actitud o práctica en la afrontación de la diarrea del menor. Sub-variables: creencias (alimentación del menor en manejo de la diarrea), tradición (quién le enseñó a manejar la diarrea), a quién acude para el tratamiento (médico, tegua o sobandero), costumbres en la prevención (consumo de agua, desparasitación del menor, lavado de manos)

- Variables Independientes: identificación de signos de hidratación, nivel de escolaridad del cuidador o madre, estrato, número de episodios diarreicos por año en el menor de 5 años, número de deposiciones por episodio diarreico en el menor en 5 años. Variables intervinientes: peso del niño, edad del niño, talla del niño, estado nutricional del niño, edad de la madre o cuidador, condiciones psicosociales, número de consultas por urgencias.

La información se recolectó por medio de la encuesta Conductas, Actitudes y Prácticas en Diarrea, aplicada mediante contacto personal con el cuidador del menor en la vivienda seleccionada. El instrumento para la recolección de información fue elaborado por la autora, en concordancia con los objetivos propuestos para el estudio. Se estructura en tres partes y está compuesto por 22 ítems que incluyen preguntas cerradas de respuesta única e ítems abiertos para la recolección de la información del menor. 
La validación se realizó en dos fases: la primera por parte de expertos y la segunda mediante prueba piloto aplicada a 15 madres y cuidadores de niños menores de 5 años, ubicadas en un sector no seleccionado para la muestra, evitando así la posible contaminación del proceso final de recolección de la información. Los expertos no sugirieron cambios en el instrumento; sin embargo, como resultado de la prueba piloto, se reformularon los enunciados correspondientes a las alternativas de respuesta de las acciones a realizar con respecto a la alimentación del menor durante el episodio de diarrea, a fin de que no hubiese ambigüedad en el momento de seleccionar la opción de respuesta.

Para el análisis de la información se recurre a métodos del análisis univariado y del análisis multivariado de datos. En el primer caso se obtienen estadísticas descriptivas básicas $(32,33)$; en el segundo, se utiliza la minería de datos y el análisis de correspondencia que permite establecer afinidades entre las modalidades de las variables categóricas (34). Este procedimiento se basa en que la nube de datos multivariados se proyecta en el plano cartesiano que maximice la varianza, identificando dos dimensiones.

Definida la orientación de los ejes de proyección del elipsoide correspondiente, se ubican las modalidades de las variables mediante la estimación de sus coordenadas en los ejes ortogonales determinados (35). Seguidamente, se establecen similitudes entre aquellas que obtengan mayores cosenos directores, esto es, que tengan los mayores valores para el coseno del ángulo conformado por los vectores directores, anclados en el origen, que determinan la ubicación de la modalidad de la variable (36).

La investigación se realizó de acuerdo con la reglamentación ética vigente, Declaración de Helsinki, Resolución No. 8430 de 1993 (37). El proyecto se clasifica como una investigación con riesgo mínimo, de acuerdo con el literal b del artículo 11 de la Resolución No. 8430 de 1993 (37), del Ministerio de Salud de Colombia.

\section{OBJETIVOS}

\section{Objetivo general}

Determinar las creencias de la madre y del cuidador sobre el manejo de la enfermedad diarreica aguda en menores de 5 años de edad en la ciudad de Bucaramanga en el año 2015.

\section{Objetivos específicos}

- Describir las características socio-demográficas de la población objeto de estudio.

- Describir las creencias y costumbres de la madre o el cuidador en el manejo del curso natural de EDA en menores de cinco años.

- Establecer la relación entre las creencias y costumbres de la madre o el cuidador en el manejo del curso natural de EDA en menores de cinco años y los factores sociales y culturales asociados.

\section{RESULTADOS}

\section{Caracterización demográfica de los cuidadores}

En cuanto a las características sociodemográficas de los cuidadores de menores de 5 años se pudo identificar que el $12 \%$ son menores de edad, el $83 \%$ son de género femenino -del cual el $65 \%$ corresponde a la madre-, sólo un $7 \%$ tiene estudios superiores, hay un $1 \%$ que manifiesta no tener estudios, el 43 $\%$ tiene estudios a nivel de primaria y los restantes bachillerato. El $73 \%$ tiene un menor para cuidar, el $24 \%$ cuida dos menores y sólo el $3 \%$ está a cargo de tres menores. El nivel socioeconómico, variable controlada, se estableció por el estrato del predio donde mora el cuidador y se seleccionó de forma tal que guardase igual proporción al de la población de Bucaramanga.

\section{Información del menor}

La muestra quedó conformada por madres o cuidadores de $54 \%$ niñas y $46 \%$ niños. Hay 50 menores de un año (27 niñas y 23 niños); 36 con edad de un año (17 niñas y 19 niños); 40 de dos años (23 niñas y 17 niños); 36 de tres años (22 niñas y 14 niños) y 38 de cuatro años (19 niñas y 19 niños).

Los promedios de pesos y tallas por grupos de edad representan variabilidad relativa no superior al 20 $\%$, medida por el coeficiente de variación; se aprecia que no difieren significativamente de los estándares nacionales.
ISSN 1794-9831

E-ISSN 2322-7028

Vol. 12 No. 2

Jul - Dic 2015

Cúcuta, Colombia 
ISSN 1794-9831

E-ISSN 2322-7028

Vol. 12 No. 2

Jul - Dic 2015

Cúcuta, Colombia

\section{Condiciones psicosociales}

En la casi totalidad de la muestra se registró que el nacimiento se había realizado en un hospital o en un puesto de salud, sólo el 1,5\% indicó que el parto se había realizado en casa. En los estratos tres a seis se encuentran menores que están asistiendo a guardería o jardín infantil en mayor proporción que en los estratos uno y dos, en una relación de 4 a 1. La mayoría de los menores que asisten a guardería o jardín son mayores de dos años, sin embargo, se encuentran menores de 2 años que asisten a guardería en los estratos uno y dos.

El $70 \%$ de las madres o cuidadores de niños(as) mayores de un año manifestó que les ha inculcado hábitos de aseo, entre los cuales está el haberle enseñado a lavarse las manos adecuadamente, encontrándose más acentuada esta costumbre en niños mayores de dos años, ya que en este grupo de edad alrededor del $90 \%$ le ha inculcado estos hábitos de higiene. Sin embargo, se encuentra que en un $60 \%$ de los casos el agua se toma directamente de la llave, solamente el $35 \%$ la hierve para el consumo y un 5 $\%$ toma agua tratada.

Con relación a esta costumbre, se encuentra que hay más casos de diarrea en menores cuando se consume el agua directamente de la llave que cuando se consume agua tratada o hervida. En el $80 \%$ de los encuestados que manifestó tomar agua directamente de la llave se presenta diarrea en el menor, mientras que esto solamente ocurre en el $50 \%$ de los que consumen agua tratada y en el $67 \%$ de los que hierven el agua para consumirla.

La desparasitación del menor se realiza en el $60 \%$ de los hogares contactados. De ellos, la gran mayoría realiza uno o dos eventos al año, el $62 \%$ desparasita al menos cada seis meses, un $28 \%$ lo realiza cada año y los restantes esporádicamente. En los encuestados que manifestaron desparasitar al menor no se han presentado más de dos episodios de diarrea al año. Por otra parte, en el $29 \%$ de los que acostumbran a desparasitar al menor y en el $46 \%$ de los que no acostumbran a desparasitarlo, no se han presentado episodios de diarrea.

\section{Deposiciones diarreicas}

El $67 \%$ de los encuestados indicó que el menor a su cargo ha tenido diarrea al menos en un episodio. De ellos, el $8 \%$ manifestó que al menor le dio diarrea una vez al año, el $48 \%$ dos veces al año y el $44 \%$ tres o más episodios de diarrea al año. En todos los casos se presentan más de cinco deposiciones por episodio diarreico. Por otra parte, el $40 \%$ acude al menos tres veces en el año a control médico por EDA, un $34 \%$ acude en dos ocasiones al año y un $16 \%$ una vez; el $10 \%$ restante no consulta al médico por EDA.

\section{Signos de deshidratación}

A los encuestados se les preguntó acerca del conocimiento de signos de deshidratación en el menor de cinco años y la mayoría conocía al menos tres signos; por ello, los resultados presentados a continuación no son mutuamente excluyentes. El 90 $\%$ coincidió en la lengua seca como principal signo de advertencia de presencia de deshidratación, un 64 $\%$ indicó la falta de orina, el $34 \%$ refirió la piel seca, un $23 \%$ hizo referencia a la ausencia de lágrimas y sólo un $7 \%$ aludió a la formación de un pliegue en la piel al tocarlo como signo de deshidratación.

En las respuestas de los cuidadores de menores con un episodio de diarrea no se aprecia diferencia significativa en los signos de deshidratación conocidos; en cambio, en aquellos en que los menores han presentado dos episodios de diarrea se observa que hay más personas que identifican la deshidratación en la falta de orina y la lengua seca que por otros signos. Por otra parte, cuando el menor ha tenido más de tres episodios de diarrea, el cuidador manifiesta que identifica la deshidratación por la ausencia de lágrimas y la falta de orina.

\section{Manejo de la diarrea y la deshidratación}

Se indagó a los encuestados acerca del manejo en el hogar de los episodios de diarrea en el menor. Las acciones más frecuentemente utilizadas por madres y cuidadores consisten en aumentarle el nivel de líquidos con respecto a los niveles que normalmente le suministra $(87 \%)$ y el aumento en el consumo de caldos (77 \%). También manifestaron que le administran sales de rehidratación oral (58\%), jugo de guayaba (55\%), sopa de arroz tostado, plátano verde o crema de pollo (46\%). El $36 \%$ le prepara y le da suero casero. En menor proporción se encontró que les daban más harina, más verduras y, en muy pocos casos, se les continúa la dieta igual. 
Al indagar por la forma de preparar el suero casero, hay coincidencia en que debe utilizarse agua, azúcar y sal, pero en la mayoría de los casos no se sabe cómo prepararlo, es decir, no se identifican las proporciones a utilizar de cada elemento para su preparación.

Se encontraron evidencias de tradición en la forma de manejar la diarrea y de mantener la hidratación, pues el $81 \%$ manifestó que un familiar (madre, abuela o tía) o un vecino les habían enseñado. También refieren las indicaciones médicas sobre el manejo de la diarrea en el $59 \%$ de los casos y en pocas proporciones indican que adquirieron información por los medios de comunicación (periódico, radio, televisión e internet). Aun cuando no lo expresan abiertamente, puede inferirse que alrededor del $38 \%$ lleva al menor donde un sobandero o tegua para el tratamiento de la diarrea.

\section{DISCUSIÓN}

Los análisis subsiguientes se realizan a partir de los resultados y se apoyan en mapas de correspondencias elaborados para comparar las modalidades de cada una de las variables categóricas involucradas en el estudio, estableciendo relaciones tanto entre costumbres y prácticas en el manejo de la diarrea en el menor por parte de sus cuidadores, como entre éstas y sus características sociales y culturales asociadas.

Uno de los aspectos importantes a discutir hace referencia a las correspondencias entre las creencias sobre el tratamiento de la diarrea en asociación con el estrato socioeconómico del cuidador (figura 1). Al establecer afinidades entre las diversas modalidades de las dos variables representadas, se pueden identificar dos grupos que se encuentran señalados en la figura; los estratos tres y cinco tienen comportamientos diferentes a los clasificados.

Se aprecia afinidad en las creencias de los estratos uno (E1) y dos (E2), quienes manifiestan asociación con la creencia de darle más líquidos de lo normal (C2) y administrarle sales de rehidratación oral (C1), presentándose esta creencia más acentuada en el estrato dos que en el uno. También se asocian con la creencia de suministrarle suero casero para el tratamiento de la diarrea (C3). El estrato tres (E3) no muestra una tendencia en cuanto a la inclinación por alguna de las creencias; al ubicarse en la parte central de la figura refleja que en él se encuentran cuidadores que se identifican con las cinco creencias analizadas, en proporciones similares.

Figura 1. Correspondencia entre estrato y creencias en manejo de EDA.

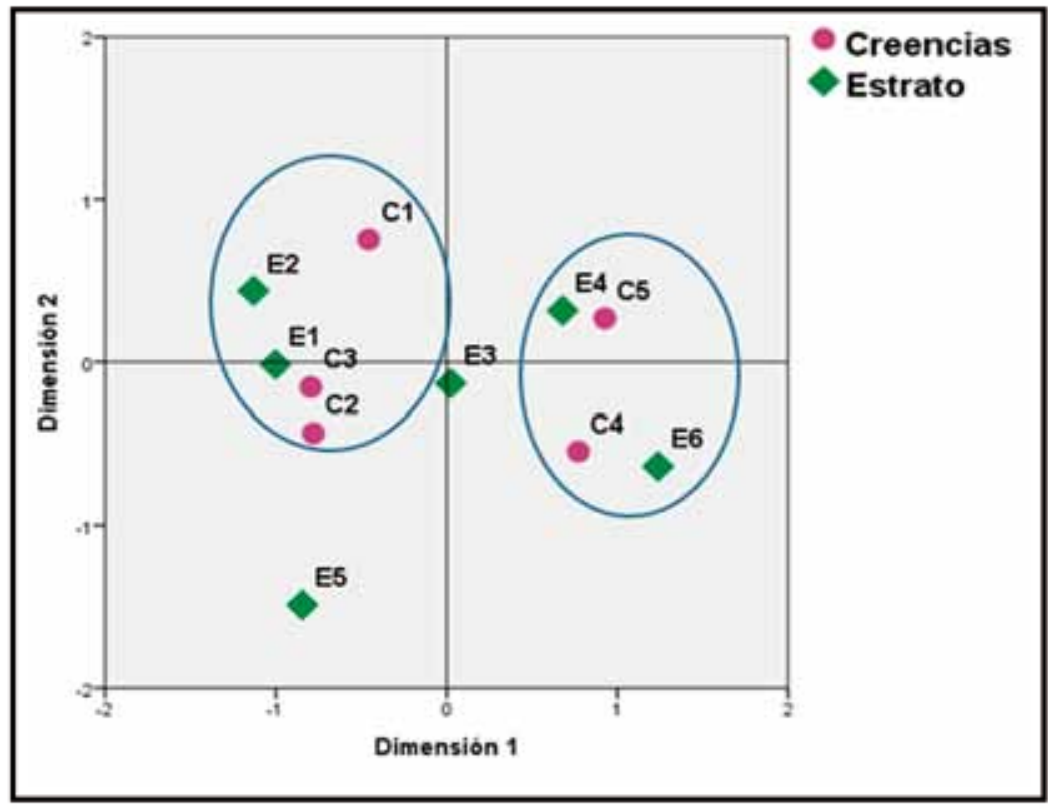

Fuente: Encuesta Conductas, Actitudes y Prácticas en Diarrea, 2015. 
ISSN 1794-9831

E-ISSN 2322-7028

Vol. 12 No. 2

Jul - Dic 2015

Cúcuta, Colombia
Ahora bien, con referencia a las creencias de suministrarle caldos (C4) y darle jugo de guayaba y sopas (C5), éstas se corresponden más con madres y cuidadores de los estratos cuatro (E4) y seis (E6), aun cuando también los del estrato seis se identifican con darles más liquido de lo normal $(\mathrm{C} 2)$ y el estrato cuatro con la administración de sales de rehidratación oral.

Las madres y cuidadores del estrato cinco (E5) presentan la creencia de tratar la diarrea con el suministro de más líquido de lo normal (C2) y del suero casero $(\mathrm{C} 3)$ con alguna inclinación por el suministro de caldo (C4) pero no manifiestan que suministren jugo de guayaba y sopas (C5).

En la figura 2 se adicionan, con respecto al análisis presentado en la figura 1, variables psicosociales para el análisis de correspondencias; su inclusión modifica la variabilidad de la muestra y, por ende, la asociación entre las diferentes modalidades de cada una de las variables. Bajo estas nuevas variables, se pueden identificar tres grupos afines.
Se aprecia que en los estratos uno y dos -ubicados a la derecha del diagrama-, es donde más se consume agua directamente de la llave (A1). También allí es donde se encuentran más cuidadores que manifiestan no haberle enseñado al menor el lavado adecuado de manos y puede inferirse que no acostumbran a desparasitar al menor; en estos estratos prima la costumbre de administrar sueros caseros a los menores durante los episodios de diarrea.

Un segundo grupo lo conforman los cuidadores de los estratos tres y cuatro. Ellos manifiestan preferencias por consumir agua hervida (A2) y también afirman que les han enseñado el lavado adecuado de manos a los menores que cuidan, pero, por lo general, no acostumbran a desparasitar a los niños. Para el tratamiento de la diarrea lo que más utilizan es la administración de sales de hidratación oral, les dan a los menores más líquidos de lo normal y les proporcionan jugo de guayaba y sopas.

Figura 2. Correspondencia entre costumbres por estrato y creencias en manejo de EDA.

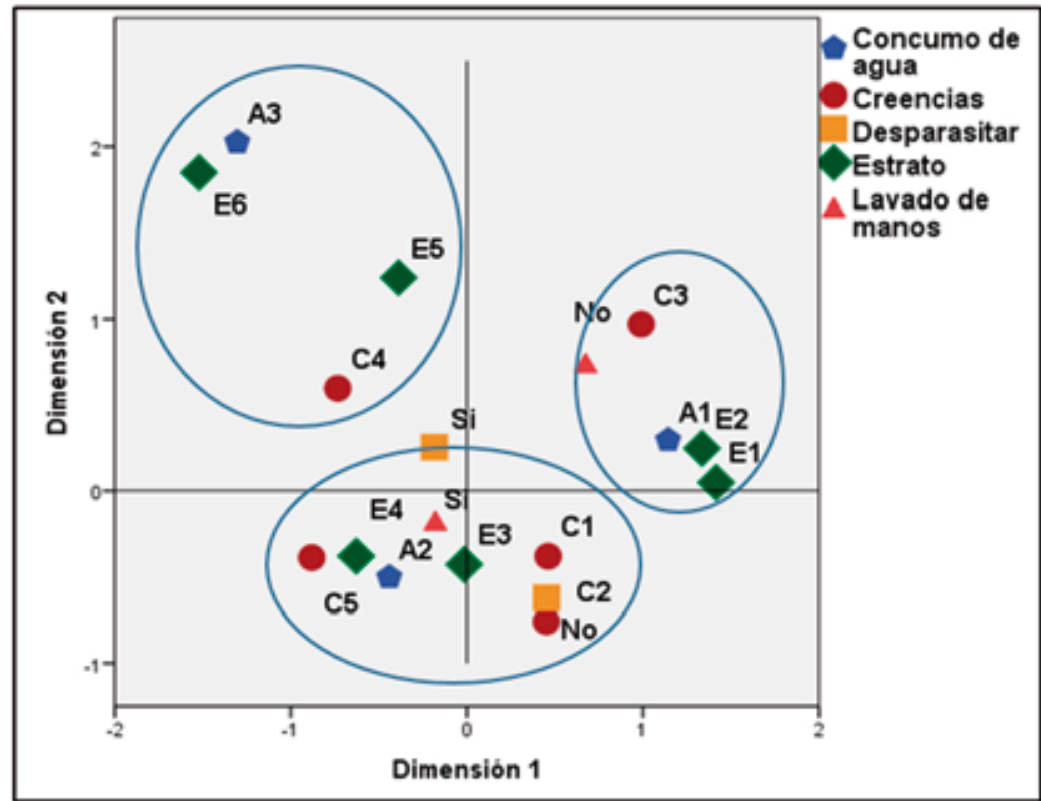

Fuente: Encuesta Conductas, Actitudes y Prácticas en Diarrea, 2015. 
El tercer grupo lo constituyen los cuidadores de los estratos cinco y seis, que se caracterizan por ser aquel en el que más se consume el agua tratada y, durante el episodio de diarrea, le suministran caldos a los menores y, en menor proporción, suero casero, jugo de guayaba y sopas.

La costumbre de desparasitar a los menores se ubica cerca al cruce de los dos ejes, lo cual permite inferir que esta costumbre es común en las madres y cuidadores de todos los estratos, acentuándose más su utilización en los estratos cinco y seis.

En la figura 3 se establecen relaciones entre la tradición en las costumbres para el tratamiento de la diarrea en menores de cinco años y las tradiciones en las costumbres ancestrales. Con la inclusión de estas variables se identifica dos grupos.
Un primer grupo lo integran los estratos uno, dos y tres; en él lo predominante es que las personas que le enseñaron al cuidador a manejar la diarrea fueron la abuela y la tía, y, en menor proporción, los vecinos, amigos y la madre. Los cuidadores manifiestan que han acudido al sobandero como parte del tratamiento de la diarrea en el niño(a) y se aprecia una tendencia hacia la costumbre de no desparasitar al menor.

El segundo grupo está conformado por las madres y cuidadores de los estratos cuatro, cinco y seis. En él se evidencia la costumbre de desparasitar al menor y se acentúa la tendencia de no acudir al sobandero como parte del tratamiento de la diarrea. Se encuentra que es la madre quien les ha enseñado acerca del tratamiento de la diarrea, seguida por la tía y, en menor proporción, los vecinos y amigos.

Figura 3. Correspondencia entre costumbres por estrato y tradición en manejo de EDA.

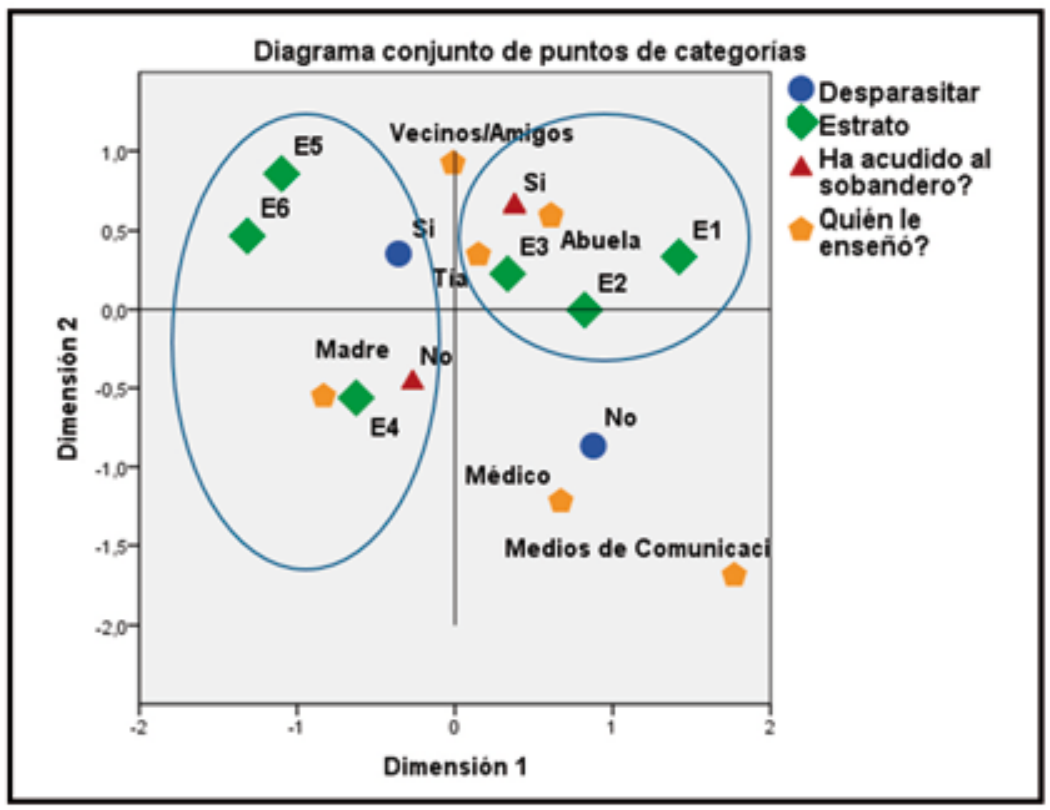

Fuente: Encuesta Conductas, Actitudes y Prácticas en Diarrea, 2015.

La tendencia general en todos los estratos es que las costumbres sobre el manejo de la diarrea y la hidratación del menor provienen de los familiares (abuela, madre, tía) así como de los vecinos y amigos; son pocos los casos en los que se evidencia que estas tradiciones son trasmitidas por el médico o por los medios de comunicación.
En la figura 4 se relacionan las consultas al médico por enfermedad diarreica aguda (EDA), la costumbre de acudir con el menor al sobandero ante un episodio de diarrea, las creencias en el manejo de la diarrea en el hogar y la información sobre la forma que tienen los cuidadores de identificar los signos de deshidratación, junto con las variables estrato socioeconómico y el nivel de escolaridad del cuidador. Con la inclusión de estas variables se identifican tres grupos.
ISSN 1794-9831

E-ISSN 2322-7028

Vol. 12 No. 2

Jul - Dic 2015

Cúcuta, Colombia 
ISSN 1794-9831

E-ISSN 2322-7028

Vol. 12 No. 2

Jul - Dic 2015

Cúcuta, Colombia
Un primer grupo está formado por cuidadores de los estratos tres (E3) y cinco (E5). En ellos se encuentra un mayor número de cuidadores con nivel de escolaridad primaria (NE2), quienes manifiestan haber acudido al sobandero con sus menores durante los episodios de diarrea, la tendencia manifiesta es a identificar la deshidratación por la lengua seca del menor (D2) y por la formación de un pliegue en la piel al tocarlo (D3).

Se encuentra asociación entre los cuidadores de este grupo y las costumbres de administrar suero oral (C1) y aumentar el nivel de líquidos (C2) como acciones para el manejo de la diarrea en el hogar. En este grupo no se identifica una tendencia hacia el número de veces que acude al médico por consulta de EDA, aun cuando por afinidad en los cuadrantes I y IV puede decirse que acuden en promedio dos veces al médico por este motivo.

Un segundo grupo identificado lo integran cuidadores en su mayoría de estrato cuatro (E4) con niveles de escolaridad bachiller (NE3), profesional (NE4) y postgrado (NE5), que acuden en promedio dos (M2) o hasta tres (M3) veces al año a consulta médica por EDA. Manifiestan, en su mayoría, no acudir al sobandero, tienden a identificar la deshidratación por la falta de orina en el menor (D4) y sus principales costumbres para el manejo de la diarrea en el hogar son suministrarle caldos (C4) y jugos de guayaba y sopas (C5).

Figura 4. Correspondencia entre variables sociales y costumbres en manejo de EDA.

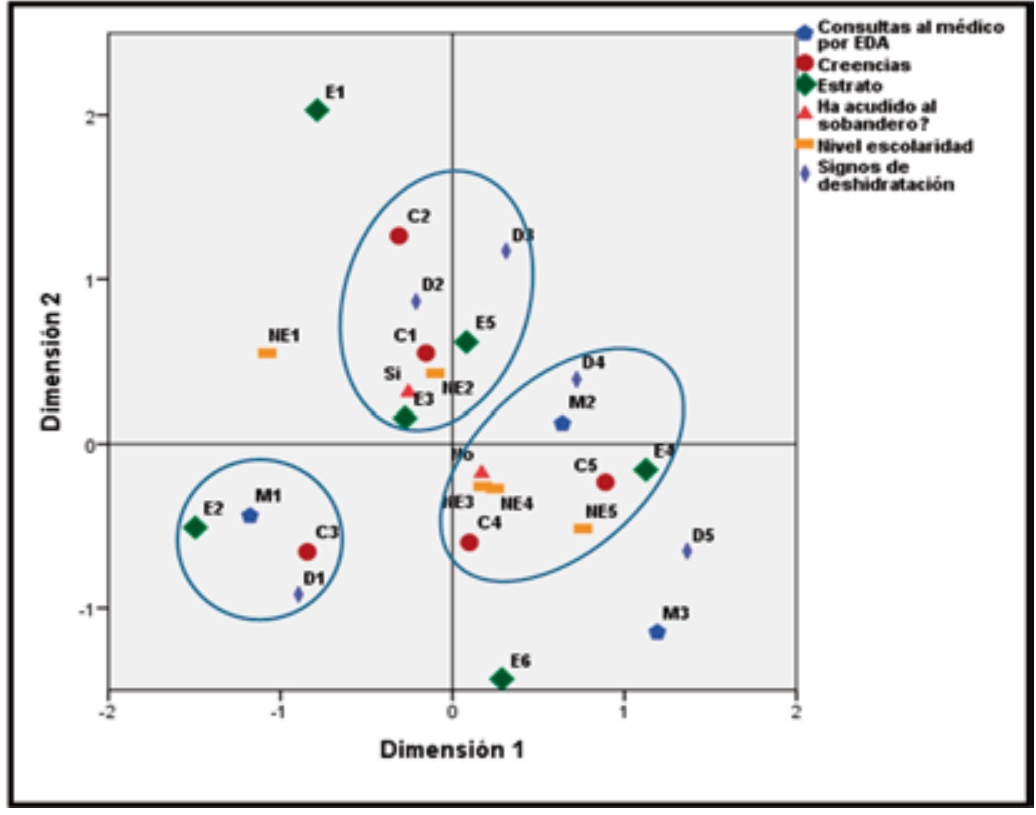

Fuente: Encuesta Conductas, Actitudes y Prácticas en Diarrea, 2015.

Un tercer grupo está conformado por cuidadores en su mayoría del estrato dos (E2) que manifiestan acudir una vez al año a consulta médica por EDA (M1), con tendencia a identificar la deshidratación por la ausencia de lágrimas $\mathrm{y}$, en menor grado, por la piel seca (D5); constituyen el grupo en donde más se acostumbra a suministrar suero casero (C3) a los menores para el manejo de la diarrea, aun cuando también se aprecia que acostumbran a suministrarle caldos (C4).
Los cuidadores de los estratos uno (E1) y seis (E6) no se encuentran dentro de alguno de los tres grupos referidos. Los del estrato uno se asocian a bajo nivel educativo, no tienen estudios (NE1) o tienen sólo primaria (NE2). Aunque no hay una asociación definida, se pueden relacionar lengua seca (D1) y pliegue en la piel (D2) como signos de identificación de diarrea y, en cuanto a creencias para el manejo de la diarrea, el suministro de más líquido de lo normal y la administración de suero casero, puede decirse que 
ellos también acostumbran a consultar al sobandero durante los episodios de diarrea.

Las madres y cuidadores del estrato seis (E6) se asocian con la consulta al médico de tres o más veces al año por EDA y con la identificación de la deshidratación por los signos de piel seca y, en menor proporción, la ausencia de lágrimas y falta de orina. No se identifica una tendencia en cuanto a nivel escolar, pero se pueden asociar a los niveles bachillerato, profesional y postgrado; sus creencias para el manejo de la diarrea se identifican con el suministro de caldo, sopas y jugos y la administración de suero casero.

En la figura 5 se relacionan las costumbres de los cuidadores en el manejo de EDA con las variables sociales y otros factores asociados. La inclusión de nuevas variables causa movilidad en la ubicación de las modalidades de cada variable analizada. Bajo estas circunstancias, se identifican cuatro grupos naturales, dos de ellos con elementos comunes.

Figura 5. Correspondencia entre costumbres en manejo de EDA con variables sociales y otros factores asociados.

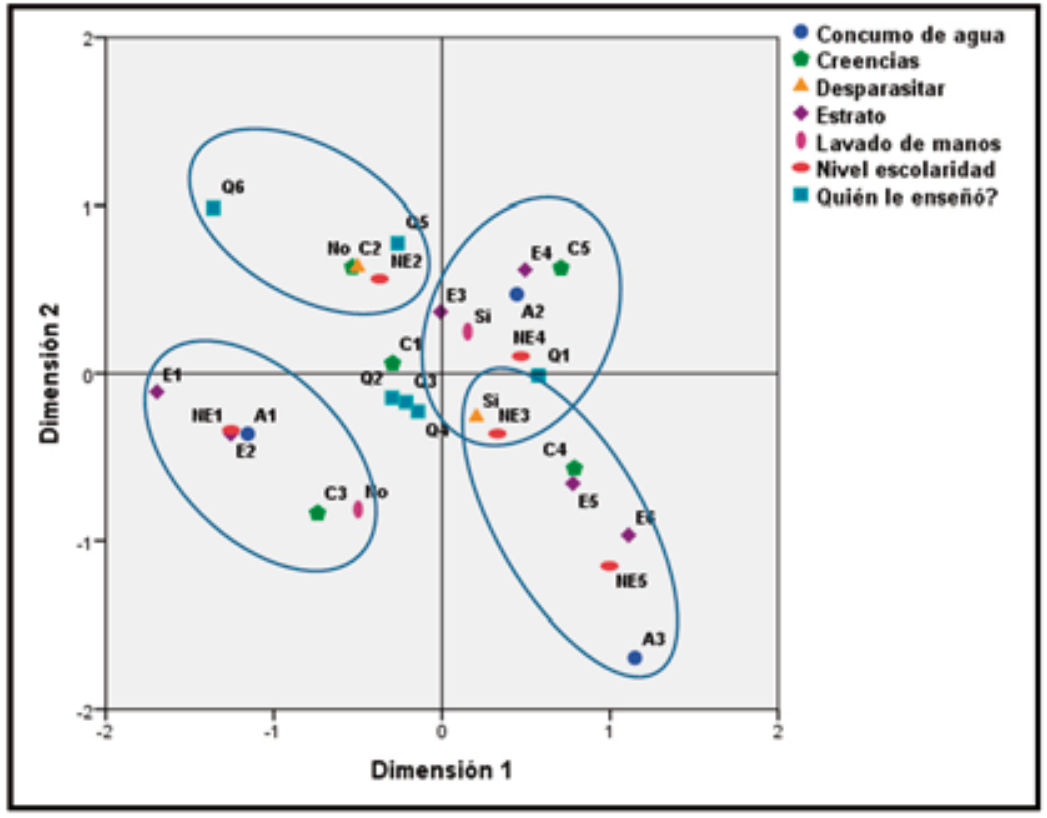

Fuente: Encuesta Conductas, Actitudes y Prácticas en Diarrea, 2015.

Un grupo está conformado por madres y cuidadores en su mayoría de los estratos uno (E1) y dos (E2) entre quienes predominan los que no tienen formación académica (NE1), no tienen la costumbre de enseñar a lavar las manos ni de desparasitar a los menores y es aquel grupo en que se encuentra un mayor número que acostumbra a tomar el agua directamente de la llave (A1). En cuanto al manejo de la diarrea predomina el suministro de suero casero (C3), el de caldos y, en menor proporción, suero oral.

Hay un grupo en el que predominan cuidadores de los estratos cinco (E5) y seis (E6), quienes acostumbran, en su mayoría, a tomar agua tratada (A3) y puede inferirse que un buen número hierve el agua para tomarla (A2), su nivel de escolaridad es de al menos profesional, acostumbran a desparasitar al menor y la costumbre predominante para el manejo de la diarrea es suministrarle caldos al niño(a) (C4). También se infiere que usan suero casero y alimentan al menor con sopas y jugos de guayaba durante el episodio $\mathrm{y}$, además, manifiestan que aprendieron de la madre sobre el manejo de la diarrea (Q1).

El siguiente grupo lo forman cuidadores en su mayoría de los estratos tres (E3) y cuatro (E4) con nivel de escolaridad bachiller o profesional, quienes tienen la costumbre de desparasitar a los menores y de enseñarles a lavarse las manos, tienen la creencia de suministrarle jugos de guayaba, sopas y caldos
ISSN 1794-9831

E-ISSN 2322-7028

Vol. 12 No. 2

Jul - Dic 2015

Cúcuta, Colombia 
ISSN 1794-9831

E-ISSN 2322-7028

Vol. 12 No. 2

Jul - Dic 2015

Cúcuta, Colombia
$(\mathrm{C} 5, \mathrm{C} 4)$ a los niños(as) durante el episodio de diarrea y prefieren hervir el agua antes de consumirla (A2), aunque hay quienes toman agua tratada. Quienes hacen parte de este grupo aprendieron sobre el manejo de la diarrea de la madre, del médico, del personal de salud y, algunos, a través de los medios de comunicación.

De igual forma, aparece un grupo en el cual no se identifica específicamente un estrato en particular, es decir, está formado por cuidadores de diferentes estratos. Allí predominan los que tienen nivel de escolaridad de primaria y aquellos que no acostumbran a desparasitar al menor a cargo; son los que acostumbran a aumentar el nivel de líquidos (C2) que le administran al menor durante la diarrea, aun cuando también utilizan suero casero y sales de rehidratación oral.

\section{CONCLUSIONES}

Los resultados muestran que la morbilidad por diarrea en menores de cinco años en Bucaramanga es de 67 $\%$, de los cuales el $90 \%$ acude a consulta médica. Se encuentra un alto porcentaje de madres y cuidadores que acuden al sobandero.

El $12 \%$ de los cuidadores de menores de 5 años está integrado por menores de edad; el $83 \%$ son de género femenino -el $65 \%$ corresponde a la madrey sólo hay un $1 \%$ que manifiesta no tener estudios. La casi totalidad de los encuestados manifestó que el nacimiento se había realizado en un hospital o en un puesto de salud; los mayores de dos años asisten a guardería.

Se observa que, en la ciudad de Bucaramanga, madres y cuidadores de menores de cinco años mantienen la tradición familiar en lo referente a identificación de signos de deshidratación y costumbres para el manejo de la diarrea. El $70 \%$ ha inculcado hábitos de aseo a los menores; sin embargo, en un $60 \%$ de los hogares el agua se toma directamente de la llave y solamente el $35 \%$ la hierve para el consumo. Por otra parte, la desparasitación del menor se realiza en el $60 \%$ de los hogares estudiados.

Acerca de las costumbres de las madres o cuidadores para el manejo de la diarrea en menores de cinco años, los datos indican que el $77 \%$ les suministra caldos, el $87 \%$ aumenta la cantidad de líquidos, el
$58 \%$ administra sales de rehidratación oral y el 55 $\%$ proporciona jugo de guayaba. Los signos más comunes de deshidratación durante los episodios de diarrea son lengua seca en el $90 \%$ de los casos, falta de orina en el $64 \%$, piel seca en el $34 \%$ y ausencia de lágrimas en el $23 \%$.

Los factores o características que hacen parte de la estructura social en la cual está inmersa la madre o el cuidador del menor, su nivel de escolaridad y las personas de quienes aprende ejercen influencia en las costumbres para el manejo de la diarrea, la consulta al médico por esta enfermedad, la ida al sobandero y la identificación de la deshidratación.

\section{CONFLICTO DE INTERESES}

El autor declara no tener ningún conflicto de intereses. 


\section{REFERENCIAS BIBLIOGRÁFICAS}

1. Farthing M, Salam M, Lindberg G, Dite P, Khalif I, Salazar E, et al. Guía Práctica de la Organización Mundial de Gastroenterología. Diarrea Aguda en Adultos y Niños: una Perspectiva Mundial. [Internet]. Reino Unido: Organización Mundial de Gastroenterología; 2012 [consultado 24 de marzo de 2015]. Disponible en: http://www. worldgastroenterology.org/UserFiles/file/guidelines/acute-diarrhea-spanish-2012.pdf

2. Ministerio de Protección Social, Organización Panamericana de la Salud (OPS). AIEPI, Libro Clínico. $2^{\mathrm{a}}$ ed. corregida y aumentada. ISBN: 978-958-8472-13-3. [Internet]. Bogotá: OPS; 2010 [consultado 24 de marzo de 2015]. Disponible en: http://es.slideshare.net/cesar7154/aiepi-libro-clinico

3. Instituto Nacional de Salud, Álvarez-Álvarez, Equipo Funcional ETA. Informe Final Mortalidad por Enfermedad Diarreica Aguda en Menores de Cinco Años y Morbilidad en Todos los Grupos de Edad, Colombia, 2014 [Internet]. Colombia: Instituto Nacional de Salud; 2014 [consultado 13 de abril de 2015]. Disponible en: http://www.ins.gov.co/ lineas-de-accion/Subdireccion-Vigilancia/Informe\%20de\%20Evento\%20Epidemiolgico/EDA\%202014.pdf

4. Instituto Nacional de Salud, Borbón-Ramos M. Mortalidad por enfermedad diarreica aguda en menores de cinco años y morbilidad en todos los grupos de edad, Colombia, año 2013. [Internet]. Colombia: Instituto Nacional de Salud; 2013 [consultado 13 de abril de 2015]. Disponible en: http://www.ins.gov.co/lineas-de-accion/SubdireccionVigilancia/Informe\%20de\%20Evento\%20Epidemiolgico/EDA\%202013.pdf

5. Instituto Nacional de Salud, Daza-Vergara JA, Grupo EDA-IRA. Informe del evento mortalidad por enfermedad diarreica aguda en menores de cinco años, y morbilidad en todos los grupos de edad, año 2012. Instituto Nacional de Salud. [Internet]. Colombia: Instituto Nacional de Salud; 2012 [consultado 13 de abril de 2015]. Disponible en: http://www.ins.gov.co/lineas-de-accion/Subdireccion-Vigilancia/Informe\%20de\%20Evento\%20Epidemiolgico/ EDA\%202012.pdf

6. Alarcón JJ, Aguirre-Cuadros E, Álvarez-Andrade EE. Conocimiento de las madres sobre la diarrea y su prevención en un asentamiento humano de la provincia de Ica. Revista Clínica de Medicina en Familia. Rev Clín Med Fam 2013; 6 (1): 25-31.

7. Tomé P, Reyes H, Rodríguez L, Guiscafré H, Gutiérrez G. Muerte por diarrea aguda en niños: Un estudio de factores pronósticos. Salud Pública Mex 1996; 38: 227-235.

8. Álvarez-Larrauri S. Maternal practices in infantile diarrheic disease and oral rehydration therapy. Salud Pública Mex 1998; 40: 256-264.

9. Keshavji II. Persistent diarrhea in Mozambican children. A study of risk factors for development of persistent diarrhoea in children aged 6 to 23 months, in Magude district, Mozambique. [Tesis en Internet]. Oslo: University of Oslo; 2003 [consultado 24 de junio de 2015]. Disponible en: https:/www.duo.uio.no/bitstream/handle/10852/30081/ keshavji.pdf?sequence $=1 \&$ is Allowed $=\mathrm{y}$

10. Fullana AM, Artero A, Sales C, Mir B, Morales M. Infantile diarrhea: knowledge and attitudes of mothers at a primary care center. Aten Primaria 1996; 17: 284-287.

11. Yalew, E. A qualitative study of community perceptions about childhood diarrhea and its management in Assosa District, West Ethiopia. BMC Public Health [Internet]. 2014 [consultado 10 de mayo de 2015]; 14:975. Disponible en: http://www.biomedcentral.com/1471-2458/14/975

12. Bruce N, Pope D, Arana B, Shields C, Romero C, Klein R, et al. Determinants of Care Seeking for Children With Pneumonia and Diarrhea in Guatemala: Implications for Intervention Strategies. American Journal of Public Health. 2014; 104(4): 647-657.

13. Arvelo W, Degollado J, Reyes L, Álvarez A. Perceptions regarding oral rehydration solutions for the management of diarrhea in Guatemalan children: implications for diarrheal management in the Americas. Pan American Journal of Public Health. 2013; 34(2): 121-126.

14. Pantenburg B, Ochoa T, Ecker L, Ruiz J. Feeding of Young Children during Diarrhea: Caregivers' Intended Practices and Perceptions. The American Society of Tropical Medicine and Hygiene. 2014; 91(3): 555-562.

15. Carter E, Bryce J, Perin J, Newby H. Harmful practices in the management of childhood diarrhea in low- and middleincome countries: a systematic review. BMC Public Health [Internet]. 2015 [consultado 10 de agosto de 2015]; 15:788. Disponible en: http://www.biomedcentral.com/1471-2458/15/788

16. Kung'u J, Owolabi O, Essien G, Aminu F, Ngnie-Teta I, Neufeld L. Promotion of Zinc Tablets with ORS through Child Health Weeks Improves Caregiver Knowledge, Attitudes, and Practice on Treatment of Diarrhoea in Nigeria. Journal of Health, Population \& Nutrition. 2015; 33(1): 9-19.

17. Lamberti L, Fischer C, Taneja S, Mazumder S, Black R. The Influence of Episode Severity on Caregiver Recall, Careseeking, and Treatment of Diarrhea among Children 2-59 Months of Age in Bihar, Gujarat, and Uttar Pradesh, India. The American Society of Tropical Medicine and Hygiene. 2015; 93(2): 250-256. 
ISSN 1794-9831

E-ISSN 2322-7028

Vol. 12 No. 2

Jul - Dic 2015

Cúcuta, Colombia
18. Profamilia. Salud sexual y reproductiva: Encuesta Nacional de Demografía y Salud. Bogotá: Profamilia; 2000.

19. Profamila. Encuesta Nacional de Demografía y Salud. Bogotá. 2011.

20. Organización Mundial de la Salud, Organización Panamericana de la Salud. Manual de atención integrada a las enfermedades prevalentes de la infancia. Washington, DC: OMS/OPS; 2012.

21. Rodríguez F, Santos C, Talani J, Tovar MF. Prácticas y creencias culturales acerca del cuidado de niños menores de un año en un grupo de madres de Chocontá, Colombia. Revista Colombiana de Enfermería. 2013; 9: 77-87.

22. Parada-Rico DA. Conocimientos, actitudes y prácticas del cuidador del menor de cinco años. Rev. cienc. cuidad 2011; 8(1): 29-39.

23. Secretaría de Salud y Ambiente de Bucaramanga, Cáceres FM, Rivero C, Parra LM, Gáfaro A, Vera LM. Análisis de la Situación de Salud. [Internet]. Bucaramanga: Secretaría de Salud y Ambiente de Bucaramanga; 2012 [consultado 24 de mayo de 2015]. Disponible en: http://www.concejodebucaramanga.gov.co/descargas/CONTROL_POLITICO_1_ SALUDAMBIENTE_2013.pdf

24. Uribe-Yepes MA, Rodríguez-Villamizar LA, Gómez-González YA, Olaya-Gamboa LE, Rodríguez-Santamaría SM. Aislamientos de patógenos comunes asociados con enfermedad diarreica aguda en menores de cinco años, Bucaramanga, Colombia. MedUNAB 2009; 12:74-79.

25. Abreu-Peralta $P$, Baracaldo-Campo HA, Robles-Carreño MI, Ochoa-Vera ME. Conocimientos, Creencias y Prácticas en el cuidado de la diarrea aguda en hogares de la zona norte de Bucaramanga, Santander 2013. ResearchGate [Internet]. 2013 [consultado 13 de abril de 2015]. Disponible en: http://www.researchgate.net/publication/258589981_ Conocimientos_Creencias_y_Prcticas_en_el_Cuidado_de_la_Diarrea_Aguda_en_Hogares_de_la_Zona_Norte_de Bucaramanga_Santander_2013

26. Hernández S, Fernández C, Baptista P. Metodología de la Investigación. México: McGraw Hill; 2010.

27. Villa A, Moreno L, García G. Epidemiología y Estadística en Salud Pública. México: McGraw Hill; 2012.

28. Bonita L, Beaglehole R, Kjellström, T. Basic Epidemiology. 2a ed. [Internet]. China: World Health Organization; 2006. [consultado 24 de mayo de 2015]. Disponible en: http://apps.who.int/iris/bitstream/10665/43541/1/9241547073_eng. pdf

29. Rothman K. Epidemiología Moderna. Madrid: Ediciones Días santos; 1987.

30. Hurtado-de Barrera J. Metodología de la investigación, guía para la comprensión holística de la ciencia. Caracas: Quirón Ediciones; 2010.

31. Hurtado I, Toro J. Paradigmas y métodos de investigación. Valencia, Venezuela: Episteme Consultores Asociados; 1997.

32. Pérez C. Métodos Estadísticos Avanzados con SPSS. Madrid: Thompson Editores; 2005.

33. Pardo A, San Martín R, Ruíz M. Metodología de las Ciencias del Comportamiento y de la Salud. Madrid: Síntesis; 2010.

34. Nisbet R, Elder J, Miner G. Handbook of statistical analysis and data mining applications. Canadá: Academic Press; 2009.

35. Dallas J. Métodos Multivariados Aplicados al Análisis de Datos. México: Thompson Editores; 2000.

36. Härdle W, Simar L. Applied Multivariate Statistical Analysis. Berlin: Springer; 2007.

37. República de Colombia, Ministerio de Salud. Resolución 8430 de 1993, octubre 4, por la cual se establecen las normas científicas, técnicas y administrativas para la investigación en salud. Diario Oficial No. 46.506. Bogotá: El Ministerio; 1993. 